\title{
Management of Type 2 Diabetes in Frail and Elderly Patients
}

\author{
Rajeev Kumar ${ }^{1 *}$ and W S Wassif ${ }^{2}$ \\ ${ }^{1}$ Consultant Physician and Endocrinologist, Bedford Hospital NHS Trust, UK \\ ${ }^{2}$ Consultant Physician, Clinical Biochemistry, Bedford Hospital NHS Trust, UK
}

Submission: March 06, 2018; Published: April 30, 2018

*Corresponding author: Rajeev Kumar, Department of Diabetes and Endocrinology, Bedford Hospital NHS Trust, Bedford MK42 9DJ, UK, Email: rajeev.kumar@bedfordhospital.nhs.uk

\begin{abstract}
The prevalence of type 2 diabetes in the old, frail individuals is rising rapidly with ageing population. Management of diabetes in these individuals is often very challenging due to a variety of features unique to this population. Most of the national and international guidelines are produced based on evidence generated from randomised controlled trials which specifically excluded old, frail patients and the evidence generated may therefore not apply to them. These patients often have a limited life expectancy and treatment aim should therefore be to keep them symptom free, and achieve a target which is realistic, while avoiding the risk of adverse effects such as hypoglycaemia.

Keywords: Diabetes mellitus; Oral antidiabetic drugs; Elderly; Frail

Abbreviations: HbA1c: Glycated Haemoglobin; CVD: Cardiovascular Disease; eGFR: Estimated Glomerular Filtration Rate, DDPP4: Dipeptidyl Peptidase 4; GLP1: Glucagon-like Peptide-1; SGLT2: The Sodium/Glucose Cotransporter 2; TZDs: Thiazolidinediones; T2DM: Type 2 Diabetes
\end{abstract} Mellitus

\section{Introduction}

Patients with type 2 diabetes suffer from hyperglycaemia due to a combination of insulin resistance and progressively declining insulin secretion as a result of loss of beta cell mass [1]. T2DM is a major risk factor for adverse cardiovascular events due to its association with obesity, lack of exercise, hypertension and dyslipidaemia. It is also associated with a variety of microvascular complications and adversely affect patients quality of life [2].

A number of guidelines have been produced across the world to help optimise management of diabetic patients and, although these guidelines apply to all adults aged $>18$ years, it may not be advisable to apply many of these guidelines for the management of old, physically frail individuals who have some features unique to them that require special consideration. The national guidelines are developed based on evidence gathered from large randomised controlled trials, which often exclude patients who are old and physically frail.

\section{Why is it important to consider frail, older adults as a separate group?}

Physical frailty is characterized by reduced ability to perform activities of daily life leading to increased dependency on others, and is associated with weight loss, reduced muscle strength, endurance and increased exhaustion [3]. Old, frail patients usually have other complex co-morbid conditions that make them particularly vulnerable to the effects and side effects of medicines. They often are on a number of other medications with the potential to trigger drug interactions leading to further complexities in the management.

Many of the older adults living in nursing homes are typically very frail. In a National Nursing Home Survey conducted in the US in 2004 diabetes was found to be highly prevalent, affecting up to $25 \%$ of residents older than 65 years and these patients had increased odds of several unfavourable outcomes [4]. It is therefore important to consider and apply specific therapeutic strategies to minimise the potential for harm while providing maximum benefit from the treatment.

\section{Is tight glycaemic control beneficial in old, frail people?}

UK Prospective Diabetes Study (UKPDS) - a trial of newly diagnosed type 2 diabetic patients with a median age 54 years [5] - advocated tight glycaemic control in order to reduce the risk of microvascular complications although it has now been called into question since the publication of Action to Control Cardiovascular Risk in Diabetes (ACCORD) trial (mean age 62.2 years) demonstrating that tight glycaemic control does have the potential to cause harm [6]. This is particularly important and relevant in the context of managing diabetes in old, frail patients 
who have relatively short life expectancy and are likely to come to serious harm should they suffer hypoglycaemic episodes due to medications [7]. Two more trails -the Action in Diabetes and Vascular Disease: Preterax and Diamicron MR Controlled Evaluation (ADVANCE) [8] and the Veterans Affairs Diabetes Trial (VADT) [9] were also published around the same time. All these trials were designed to assess the role of tight glycaemic control in preventing CVD events in high-risk patients.

However, it is important to bear in mind that none of these trials enrolled old, frail individuals. The UKPDS enrolled relatively young newly diagnosed diabetics; ADVANCE, ACCORD, and the VDAT enrolled older, but not frail, individuals (mean age 60 to 66 years) with well-established T2DM and at higher cardiovascular risk than the UKPDS patients. Moreover, while it took more than 6 years in UKPDS to accrue microvascular benefits, it took only 2 year in ACCORD trial before an increased mortality due to tight glycaemic control was observed leading to premature termination of intensive glucose lowering arm. Importantly, increased mortality was not linked to a specific drug but was due to the strategy of tight glycaemic control.

Old, frail individuals are likely to have shorter life expectancy and if these results are applied to them, they are more likely to suffer harm than any benefit they are likely to gain due to long time it takes to accrue benefits. Microvascular benefits that were observed in UKPDS are perhaps not very relevant to old, frail population and do not provide an improved quality of life which is what is more important at a ripe age. For example, there was no evidence of improved visual acuity in UKPDS with tight glycaemic control despite reduced need for laser anticoagulation. Similarly there was no decreased rate of end stage renal disease needing dialysis despite reduced microalbuminuria level in patients in tight glycaemic control arm.

It is therefore clear that tight glycaemic control with the aim to provide microvascular benefits can be harmful for old, frail population especially if it is achieved with the drugs that have the potential to induce hypoglycaemia and any benefit gained are almost inconsequential due to time frame involved and the nature of benefit accrued.

When ACCORD, ADVANCE and VADT were performed, newer generation of anti-glycaemic drugs (DDPP4 inhibitors, GLP1 agonists and SGLT2 inhibitors) were not available and glycaemic control strategies were based on metformin, sulfonylureas, TZDs and insulins. New classes of drugs that have since emerged have a much lower risk of hypoglycaemia even in older patients. It however remains to be seen if these newer drugs will confer additional clinical benefits in old, frail people.

\section{Potential harm due to risk associated with tight glycaemic control}

All randomised controlled trials have demonstrated an increased risk of hypoglycaemia with tight glycaemic control $[6,8,9]$. Moreover, older frail patients may not perceive and respond so well to hypoglycaemic episodes [7]. They often have underlying cognitive impairment with inconsistent and poor oral intake. They are more likely to suffer harm due to a hypoglycaemic episode due to risk of falls [10] and fracture and are at a higher risk of hospitalization and loosing independence. In a prospective study of 783 older adults with T2DM (mean age 74 years) it was found that the risk for developing dementia was 2-fold higher in those who experienced a hypoglycaemic event compared with those who did not have a hypoglycaemic event and the patients who developed dementia had a 3 times higher risk for having further hypoglycaemic events compared to those who did not develop dementia [11].

\section{Potential harm due to risk associated with poor glycaemic control}

A poor diabetes control can also be detrimental to the health and quality of life of older, frail individuals due to symptoms often associated with it. Patient may become polyuric and incontinent and develop dehydration and fatigue. Persistently poor control is likely to increase the risk of infection and poor wound healing. Many of the symptoms relating to poor diabetes control may in fact be attributed to frailty.

However, there is some evidence suggesting that old, frail diabetic patients are able to tolerate hyperglycaemic state better than younger patients. In a study of old diabetic patients (mean age 80 years) resident in a community nursing home, it was reported that those with an $\mathrm{HbA} 1 \mathrm{c}$ of $8.0 \%$ to $8.9 \%$ had better functional outcomes at 2 years than those with an HbA1c of $7.0 \%$ to $7.9 \%[12]$.

\section{HbA1c targets in old, frail patients with type 2 diabetes}

The American Geriatrics Society 2013 Guidelines [13] recommends a target $\mathrm{HbA} 1 \mathrm{c}$ of $8-9 \%$ for older adults with multiple comorbidities, poor health, and limited life expectancy. The American Diabetes Association 2018 Standards of Medical Care in Diabetes [14] advise that those with multiple coexisting chronic illnesses, cognitive impairment, or functional dependence should have less stringent glycaemic goals $\mathrm{HbA} 1 \mathrm{c}$ $<8.0-8.5 \%$ [64-69 mmol/mol]). These guidelines do indicate the need to relax and individualise glycaemic targets for frail, older diabetics with limited life expectancy but with no definitive recommendations.

The DCPNS/PATH guidelines, on the other hand, support the notion that HbA1c should be kept above $8 \%$ with the explicit aim to avoid hypoglycaemia and extreme hyperglycaemia [15]. It is also suggested that patients who have had high $\mathrm{HbA1c}$ for many years and have remained asymptomatic and have limited life expectancy should not have their therapy altered with a view to tighten their diabetes control.

\section{Newer generation of antiglycamic drugs}

Three new classes of antiglycamic drugs are now available - DDP4 inhibitors, GLP1 agonists and SGLT2 inhibitors. They 
all have a much lower risk of inducing hypoglycaemic events. Several randomized controlled trails such as EMPAREG [16], LEADER [17], SUSTAIN-6 [18] and CANVAS [19] with these newer agents have demonstrated cardiovascular and mortality benefits in patients with long-standing diabetes and high cardiovascular risk (mean age of patient 63-65 years and mean duration of follow up 2-4 years) treated to achieve an HbA1c of $<7 \%$. Whether the results can be generalized to older, frailer patients is difficult to assess.

\section{Anti-hyperglycaemic drugs to use in old, frail type 2 diabetic patients}

Management of diabetes among older frail adults can be challenging due to heterogeneity and varying needs in this population and is often compounded by their inconsistent and poor oral intake with less than satisfactory compliance with diet regime. One should take especial care while prescribing medications to these patients. The treatment regimen should be kept simple bearing in mind the possibility of drug interactions and side effects. Many of these patients may also be taking medications that are not on regular prescription such as vitamins, minerals, herbal supplements etc.

Metformin, in view of its low cost and very low hypoglycaemia risk, should be the preferred first-line agent in combination with lifestyle therapy [20]. It should however not be used in patients with an eGFR of less than $30 \mathrm{~mL} / \mathrm{min}$ per $1.73 \mathrm{~m}^{2}$. A lower dose with more frequent monitoring of renal functions may be appropriate for individuals with an eGFR between 30 and 60mL/ min per $1.73 \mathrm{~m}^{2}$.

Sulfonylureas, especially glyburide, are generally avoided in this patient population due to inconsistent oral intake and risk of hypoglycaemia. However, in patients with high HbA1c the risk of hypoglycaemia is rather low and it should be possible to use them with caution and some degree of blood glucose monitoring. Short-acting drugs, such as glipizide, may be more appropriate [21]. TZDs, unless contraindicated due to underlying other comorbidities such as congestive cardiac failure or bladder cancer, are also appropriate in this patient population due to their low cost and low risk of hypoglycaemia. They can also be used in patients with impaired renal functions. About $5 \%$ patients on this class of drugs may develop peripheral oedema during treatment [22].

DDP4 inhibitors or "gliptins" are a very attractive option for old frail individuals as they have a low risk of hypoglycaemia and gastrointestinal side effects and are administered once daily [23]. They are however relatively less efficacious compared to other classes of anti-glycaemic drugs. They have a very favourable safety profile and can be combined with basal insulin to keep the regimen simple. All DPP4 inhibitors, except Linagliptin, require dose adjustment in those with impaired renal functions. GLP-1 agonists are injectable drugs, which are more efficacious than gliptins and carry only a very small risk of hypoglycaemia and are generally well tolerated [23]. Newer formulations are administered once weekly and may be suitable for those with high pill burden. They might be beneficial in a select group of old patients who are obese and where substantial HbA1c lowering is necessary. They should not be used in patients with impaired renal functions.

SGLT-2 inhibitors are a relatively new class of medicine and although they are administered once daily and cause weight loss and lead to blood pressure reduction they should be used cautiously especially in old patients due to risk of hypotension and urogenital infection [24]. They are not licenced for patients over 75 years of age.

Insulin may well be the drug of choice for many old, frail diabetic patients due to their underlying low BMI, renal impairment and other complex co-morbidities, which may contraindicate other oral or injectable anti-hyperglycaemic. A long acting basal insulin added to oral hypoglycaemic drugs may be sufficient to keep the patients symptom free while avoiding the risk of hypoglycaemia that may be associated with the use of bolus insulin especially in those with unpredictable oral intake [25]. Patients with visual problems should be able to administer certain long acting insulin, such as Insulatard and Levemir, with Innolet device which makes dialling of the dose easier.

\section{Conclusion}

Almost all national and international guidelines for the management of type 2 diabetic patients are based on evidence generated from randomised controlled trails that did not include old, frail patients and may therefore not be applicable to them. These patients are unlikely to benefit from reducing the risk of microvascular complications and are more likely to suffer harm due to increased risk of hypoglycaemia. However, patients with very poorly controlled diabetes will be at risk of hyperglycaemic complications including hyperglycaemic hyperosmolar coma. Optimising glycaemic control should therefore aim to avoid hypoglycaemic and hyperglycaemic consequences. Newer generation of antiglycamic drugs provide cardiovascular and mortality benefit relatively quickly (within 2-4 years) but whether these benefits can be extrapolated to old, frail people is not yet clear.

We, as clinicians, should work in collaboration with patients, their families, and caregivers to agree individualised glycaemic targets with the aim to avoid hypoglycaemic episodes and keep patients symptom free. Some patients, such as those with a life expectancy of more than 5 years, may benefit from more intensive glycaemic control. The treatment regimen should be kept inexpensive and simple and the targets should be clearly documents and communicated to all health care professionals involved in patient's care.

\section{References}

1. Kahn SE (2003) The relative contributions of insulin resistance and beta-cell dysfunction to the pathophysiology of type 2 diabetes. Diabetologia 46(1): 3-19. 


\section{Current Research in Diabetes \& Obesity Journal}

2. Donnelly R, Emslie Smith AM, Gardner ID, Morris AD (2000) Vascular complications of diabetes. BMJ 320: 1062.

3. Morley JE, Vellas B, van Kan GA, Anker SD, Bauer JM, et al. (2013) Frailty consensus: A call to action. J Am Med Dir Assoc 14(6): 392-397.

4. Resnick HE, Heineman J, Stone R, Shorr RI (2008) Diabetes in US nursing homes, 2004. Diabetes Care 31(2): 287-288.

5. UK Prospective Diabetes Study (UKPDS) Group (1998) Intensive blood-glucose control with sulphonylureas or insulin compared with conventional treatment and risk of complications in patients with type 2 diabetes (UKPDS 33). Lancet 352(9131): 837-853.

6. Gerstein HC, Miller ME, Byington RP, Goff DC, Bigger JT, et al. (2008) Effects of intensive glucose lowering in type 2 diabetes. N Engl J Med 358(24): 2545-2559.

7. Meneilly GS, Cheung E, Tuokko H (1994) Altered responses to hypoglycemia of healthy elderly people. J Clin Endocrinol Metab 78(6): $1341-1348$

8. Patel A, MacMahon S, Chalmers J, Neal B, Billot L, et al. (2008) Intensive blood glucose control and vascular outcomes in patients with type 2 diabetes. N Engl J Med 358(24): 2560-2572.

9. Duckworth W, Abraira C, Moritz T, Reda D, Emanuele N, et al. (2009) Glucose control and vascular complications in veterans with type 2 diabetes. VDAT Study. N Engl J Med 360(2): 129-139.

10. Nelson JM, Dufraux K, Cook PF (2007) The relationship between glycemic control and falls in older adults. J Am Geriatr Soc 55(12) 2041-2044.

11. Yaffe K, Falvey CM, Hamilton N, Harris TB, Simonsick EM, et al. (2003) Association between hypoglycemia and dementia in a biracial cohort of older adults with diabetes mellitus. JAMA Intern Med 173(14): $1300-1306$

12. Yau CK, Eng C, Cenzer IS, Boscardin WJ, Rice Trumble K, et al. (2012) Glycosylated hemoglobin and functional decline in communitydwelling nursing home-eligible elderly adults with diabetes mellitus. J Am Geriatr Soc 60(7): 1215-1221.

13. Moreno G, Mangione CM, Kimbro L, Vaisberg E, American Geriatrics Society Expert Panel on Care of Older Adults with Diabetes Mellitus (2013) Guidelines Abstracted from the American Geriatrics Society Guidelines for Improving the Care of Older Adults with Diabetes Mellitus: 2013 Update. J Am Geriatr Soc 61(11): 2020-2026.
14. American Diabetes Association: Standards of medical care in diabetes (2018) Diabetes Care. 41 (Supplement 1): S1-S2.

15. Mallery LH, Ransom T, Steeves B, Cook B, Dunbar P, et al. (2013) Evidence-informed guidelines for treating frail older Adults with type 2 diabetes: From the Diabetes Care Program of Nova Scotia (DCPNS) and the Palliative and Therapeutic armonization (PATH) Program. J Am Med Dir Assoc 14(11): 801-808.

16. Zinman B, Wanner C, Lachin JM, Fitchett D, Bluhmki E, et al. (2015) Empagliflozin, cardiovascular outcomes, and mortality in type 2 diabetes. N Engl J Med 373(22): 2117-2128.

17. Marso SP, Daniels GH, Brown Frandsen K, Kristensen P, Mann JF, et al. (2016) Liraglutide and cardiovascular outcomes in type 2 diabetes. $N$ Engl J Med 375(4): 311-322.

18. Marso SP, Bain SC, Consoli A, Eliaschewitz FG, Jódar E, et al. (2016) Semaglutide and cardiovascular outcomes in patients with type 2 diabetes. N Engl J Med 375(19): 1834-1844.

19. Neal B, Perkovic V, Mahaffey KW, De Zeeuw D, Fulcher G, et al. (2017) Canagliflozin and cardiovascular and renal events in type 2 diabetes. $\mathrm{N}$ Engl J Med 377(7): 644-657.

20. Boussageon R, Supper I, Bejan Angoulvant T, Kellou N, Cucherat M, et al. (2012) Reappraisal of metformin efficacy in the treatment of type 2 diabetes: a meta-analysis of randomised controlled trials. PLoS Med 9(4): e1001204.

21. Chanal H (2013) Should elderly patients with type 2 diabetes be treated with glibenclamide (glyburide) or different sulphonylurea? World Health Organization, Geneva, Switzerland.

22. Yki Jarvinen H (2014) Thiazolidinediones. N Engl J Med 351(11): 1106-1118.

23. Amori RE, Lau J, Pittas AG (2007) Efficacy and safety of incretin therapy in type 2 diabetes: systematic review and meta-analysis. JAMA 298(2): 194-206

24. Cefalu WT, Riddle MC (2015) SGLT2 inhibitors: the latest "new kids on the block"! Diabetes Care 38(3): 352-354.

25. Karnieli E, Baeres FM, Dzida G, Ji Q Ligthelm R, et al. (2013) Observational study of once-daily insulin detemir in people with type 2 diabetes aged 75 years or older: a sub-analysis of data from the Study of Once daily LeVEmir (SOLVE). Drugs Aging 30(3): 167-175.

Your next submission with Juniper Publishers
will reach you the below assets
- Quality Editorial service
- Swift Peer Review
- Reprints availability
- E-prints Service
- Manuscript Podcast for convenient understanding
- Global attainment for your research
- Manuscript accessibility in different formats
( Pdf, E-pub, Full Text, Audio)
- Unceasing customer service
Track the below URL for one-step submission
https://juniperpublishers.com/online-submission.php

